and one is at once recognised as a new man. I had a vivid reminder of this at a recent meeting of consultant psychotherapists called to discuss the impending changes; a colleague showed a transparency of a beleagured psychotherapy service being menaced by two resource-seeking crocodiles labelled 'social workers' and 'psychologists'. I believe it is our patients who are menaced when professional relationships are construed in such terms of "mutual hostility" cloaked under the name of "fair competition".

There is a particular irony in the use of the new language in 1990, the year in which the market has apparently been wholeheartedly embraced by Eastern Europe. In the name of supposedly libertarian market values, a system of management is being created for the National Health Service which will be instantly recognised by those from Eastern Europe: with minimal exposure to public influence or inspection and with a much reduced professional participation, a vertical command structure is being created in which personal advancement for those lower in the hierarchy can only depend upon pleasing those higher up.

The defence of our patients' interests and of our own profession demands exceptional vigilance and I believe the preservation of clear speech is crucial. In making our own proposals, in considering managements plans, and in auditing our own work we must never leave out of what is said and what is recorded the information relating to the point of our enterprise, namely the provision of health care to the people of this country. The quality of care available, the efficiency with which it reaches those in need, the costs in human terms of what is not provided and the conditions of work of the professionals providing care should all appear in every evaluation. We are in the business of providing a service, not of serving a business, and our concerns must be expressed in, and defended by, the language appropriate to our professional commitment to that service.

St Thomas' Hospital

A. RYLE

London SE1 7EH

\section{Teaching descriptive psychopathology to medical students}

Dear Sirs

Making psychiatry interesting for medical students is one of our great priorities. It is the only way we can recruit more doctors into the specialty at a time when more and more medical personnel tend to see other areas of practice as more rewarding.

Students tend to find descriptive psychopathology difficult. This is quite understandable. The terminology of psychopathology is not very precise and there are several phenomena which are easily mistaken for something else. The clarification of the concepts of descriptive psychopathology is an on-going assignment for us all.

I have discovered that by asking medical students to "Make a list of the things we do with our minds", and "Against each entry put down your own ideas of the various ways in which the function concerned can become abnormal", a good foundation is laid for the understanding of psychopathology. By the time they have worked through this activity they have, in fact, come up with descriptions of most of the signs and symptoms of mental disorder, even if they do not use the proper terminology.

A couple of tutorials clarifies their thinking about these things, as well as providing or confirming the correct terms for the signs and symptoms they have already figured out and adding those they missed.

I have found that because this exercise challenges the students' initiative and sense of discovery, it is a much more interesting activity for them than lectures on the subject.

\section{The Royal London Hospital (St Clement's)}

London E3 4LL

\section{Chaos in mind}

\section{Dear Sirs}

I would like to comment on the conference report, 'The Atom in Mind' (Psychiatric Bulletin, September $1990,14,559$ ).

With the growing interest in the behaviour of dynamic systems in medicine, the reductionalist approach may not be the way forward when addressing the mind-body problem. Pressing the techniques of the individual specialisms of psychology, neurophysiology, theology, physics and mathematics to their limits may result in further division. The study of systems and chaos, although having its origins in mathematics, tends to unify across disciplines.

The brain is a complex dynamic system, with feedback at multiple levels of organisation. It is, however, not isolated but exists in the context of many other systems: the body, the environment, the family, and society. It is within these systems that body image and self-esteem are defined. The mind may therefore exist as a product of multiple dynamic systems, interacting at different scales, creating one whole. There is, however, more than mere complexity of the holistic approach which would be beyond analysis. For example, consider the brain alone: it contains $10^{13}$ $10^{14}$ synapses each of which will display non-linear behaviour. Simple dynamic systems, however, containing few non-linear elements can have complex, seemingly stochastic and unpredictable behaviour even though traditionally one would expect deterministic and thus predictable properties. Such 